\title{
CONDITIONAL YEH-WIENER INTEGRALS WITH VECTOR-VALUED CONDITIONING FUNCTIONS
}

\author{
CHULL PARK AND DAVID SKOUG
}

(Communicated by William D. Sudderth)

\begin{abstract}
In this paper we establish various results involving conditional YehWiener integrals with vector-valued conditioning functions. We first develop a very simple formula for converting conditional Yeh-Wiener integrals into ordinary (i.e., nonconditional) Yeh-Wiener integrals. We then use this simple formula to evaluate the conditional Yeh-Wiener integral of various functionals, including functionals in which the sectional average of an $L_{2}$-function occurs naturally. Finally, we use these results to obtain a general Cameron-Martin type translation theorem for conditional Yeh-Wiener integrals.
\end{abstract}

\section{INTRODUCTION}

For $Q=[0, S] \times[0, T]$ let $C(Q)$ denote Yeh-Wiener space, i.e., the space of all real-valued continuous functions $x(s, t)$ on $Q$ such that $x(0, t)=$ $x(s, 0)=0$ for every $(s, t)$ in $Q$. Yeh [10] defined a Gaussian measure $m_{y}$ on $C(Q)$ such that as a stochastic process $\{x(s, t),(s, t) \in Q\}$ has mean $E[x(s, t)]=\int_{C(Q)} x(s, t) m_{y}(d x)=0$ for every $(s, t)$ in $Q$ and covariance $E[x(s, t) x(u, v)]=\min \{s, u\} \min \{t, v\}$. This process is called the standard Yeh-Wiener (or two-time parameter Wiener) process on $Q$.

For each partition $\tau=\tau_{m, n}=\left\{\left(s_{i}, t_{j}\right), i=1, \ldots, m, u=1, \ldots, n\right\}$ of $Q$ with $0=s_{0}<s_{1}<\cdots<s_{m}=S$ and $0=t_{0}<t_{1}<\cdots<t_{n}=T$, define $X_{\tau}: C(Q) \rightarrow \mathbf{R}^{m n}$ by $X_{\tau}(x)=\left(x\left(s_{1}, t_{1}\right), \ldots, x\left(s_{m}, t_{n}\right)\right)$. Let $\mathscr{B}^{m n}$ be the $\sigma$-algebra of Borel sets in $\mathbf{R}^{m n}$. A Yeh-Wiener interval is a set of the type

$$
I=\left\{x \in C(Q): X_{\tau}(x) \in B\right\}=X_{\tau}^{-1}(B), B \in \mathscr{B}^{m n} .
$$

The Yeh-Wiener measure $m_{y}$ of such a set is given by

$$
m_{y}(I)=\int_{B} K(\tau, \vec{\xi}) d \vec{\xi}
$$

where

$$
\vec{\xi}=\left(\xi_{1,1}, \ldots, \boldsymbol{\xi}_{m, n}\right) \in \mathbf{R}^{m n}
$$

Received by the editors April 18, 1988.

1980 Mathematics Subject Classification (1985 Revision). Primary 60J65, $28 \mathrm{C} 20$.

Key words and phrases. Yeh-Wiener integral, conditional Yeh-Wiener integral, Cameron-Martin translation theorem. 
and

$$
\begin{aligned}
& K(\tau, \vec{\xi})=(2 \pi)^{-m n / 2}\left[\prod_{i=1}^{m}\left(s_{i}-s_{i-1}\right)\right]^{-n / 2}\left[\prod_{j=1}^{n}\left(t_{j}-t_{j-1}\right)\right]^{-m / 2} \\
& \cdot \exp \left\{-\frac{1}{2} \sum_{i=1}^{m} \sum_{j=1}^{n}\left(\xi_{i, j}-\xi_{i-1, j}-\xi_{i, j-1}+\xi_{i-1, j-1}\right)^{2} /\left(s_{i}-s_{i-1}\right)\left(t_{j}-t_{j-1}\right)\right\},
\end{aligned}
$$

with $\xi_{i, 0}=\xi_{0, j}=0$.

Let $\mathscr{F}_{\tau}$ be the $\sigma$-algebra generated by the sets $\left\{X_{\tau}^{-1}(B): B \in \mathscr{B}^{m n}\right\}$ with $\tau=\tau_{m, n}$ fixed. Then, by the definition of conditional expectation (see Tucker [9] and Yeh [13]), for each Yeh-Wiener integrable function $F(x)$

$$
\begin{aligned}
\mu_{\tau}(B) & \equiv \int_{X_{\tau}^{-1}(B)} F(x) m_{y}(d x)=\int_{X_{\tau}^{-1}(B)} E\left(F \mid \mathscr{F}_{\tau}\right) m_{y}(d x) \\
& =\int_{B} E\left(F(x) \mid X_{\tau}(x)=\vec{\xi}\right) P_{X_{\tau}}(d \vec{\xi}), \quad B \in \mathscr{B}^{m n},
\end{aligned}
$$

where $P_{X_{\tau}}(B)=m_{y}\left(X_{\tau}^{-1}(B)\right)$, and $E\left(F(x) \mid X_{\tau}(x)=\vec{\xi}\right)$ is a Borel measurable function of $\vec{\xi}$ which is unique up to Borel null sets in $\mathbf{R}^{m n}$. Since $X_{\tau}^{-1}(B)$ is a Yeh-Wiener interval, it follows from (1.1) that

$$
P_{X_{\tau}}(d \vec{\xi})=K(\tau, \vec{\xi}) d \vec{\xi}
$$

Conditional expectations of the type $E\left(F(x) \mid X_{\tau}(x)=\vec{\xi}\right)$ are often called conditional Yeh-Wiener integrals, and have been studied extensively by many authors (see [2], [3], [4] and further references in these papers). Some results are obtained by Chang and Ahn [2] and Chung and Ahn [4] for conditional Yeh-Wiener integrals when $X_{\tau}(x)=x(S, T)$, i.e., when $m=n=1$.

The main purpose of this paper is to develop a useful, but rather simple formula to convert conditional Yeh-Wiener integrals into ordinary (i.e., nonconditional) Yeh-Wiener integrals, and then to use this formula to obtain a general Cameron-Martin Translation Theorem for conditional Yeh-Wiener integrals when the conditioning function is vector-valued. We also use this formula to evaluate the conditional Yeh-Wiener integral of various functions. The sectional average of an $L_{2}$-function proves useful when we consider functions of the form $F\left(\int_{Q} h_{1} d x, \ldots, \int_{Q} h_{N} d x\right)$ where each $\int_{Q} h_{i} d x$ is a stochastic integral with mean zero and variance $\left\|h_{i}\right\|_{2}^{2}$. 


\section{A USEFUL BUT SIMPLE FORMULA}

\section{FOR CONDITIONAL YEH-WIENER INTEGRALS}

For each partition $\tau=\tau_{m, n}$ of $Q$ and $x \in C(Q)$, define the quasi-polyhedric function $[x]$ on $Q$ by

$$
\begin{aligned}
{[x](s, t)=} & x\left(s_{i-1}, t_{j-1}\right)+\left[\left(s-s_{i-1}\right)\left(t-t_{j-1}\right) /\left(\Delta_{i} s \Delta_{j} t\right)\right] \Delta_{i j} x(s, t) \\
& +\left[\left(s-s_{i-1}\right) / \Delta_{i} s\right]\left(x\left(s_{i}, t_{j-1}\right)-x\left(s_{i-1}, t_{j-1}\right)\right) \\
& +\left[\left(t-t_{j-1}\right) / \Delta_{j} t\right]\left(x\left(s_{i-1}, t_{j}\right)-x\left(s_{i-1}, t_{j-1}\right)\right)
\end{aligned}
$$

on each $Q_{i j}=\left(s_{i-1}, s_{i}\right] \times\left(t_{j-1}, t_{j}\right], i=1, \ldots, m, j=1, \ldots, n$ where $\Delta_{i} s=$ $s_{i}-s_{i-1}, \Delta_{j} t=t_{j}-t_{j-1}, \Delta_{i j} x(s, t)=x\left(s_{i}, t_{j}\right)-x\left(s_{i-1}, t_{j}\right)-x\left(s_{i}, t_{j-1}\right)+$ $x\left(s_{i-1}, t_{j-1}\right)$ and $[x](s, t)=0$ if $s t=0$.

Similarly, for each $\vec{\xi}=\left(\xi_{1,1}, \ldots, \xi_{m, n}\right) \in \mathbf{R}^{m n}$, define the quasi-polyhedric function $[\vec{\xi}]$ on $Q$ by

$$
\begin{aligned}
{[\vec{\xi}](s, t)=} & \xi_{i-1, j-1}+\left[\left(s-s_{i-1}\right)\left(t-t_{j-1}\right) /\left(\Delta_{i} s \Delta_{j} t\right)\right] \Delta_{i j} \vec{\xi} \\
& +\left[\left(s-s_{i-1}\right) / \Delta_{i} s\right]\left(\xi_{i, j-1}-\xi_{i-1, j-1}\right) \\
& +\left[\left(t-t_{j-1}\right) / \Delta_{j} t\right]\left(\xi_{i-1, j}-\xi_{i-1, j-1}\right)
\end{aligned}
$$

on each $Q_{i j}$ where $\Delta_{i j} \vec{\xi}=\xi_{i, j}-\xi_{i-1, j}-\xi_{i, j-1}+\xi_{i-1, j-1}, \xi_{0, j}=\xi_{i, 0}=0$ for all $i$ and $j$, and $[\vec{\xi}](s, t)=0$ if $s t=0$.

We note that both $[x]$ and $[\vec{\xi}]$ belong to $C(Q)$ for each $x$ in $C(Q)$ and each $\vec{\xi}$ in $\mathbf{R}^{m n}$. In addition for all $i$ and $j,[x]\left(s_{i}, t_{j}\right)=x\left(s_{i}, t_{j}\right)$ and $[\vec{\xi}]\left(s_{i}, t_{j}\right)=$ $\xi_{i, j}$. Also on each $Q_{i j}$, both $[x](s, t)$ and $[\xi](s, t)$ are quadratic functions of two variables and each is a linear function of one variable for each value of the other variable. The functions $[x]$ with $m=n$ and binary partition points were used as binary quadratic approximations in [6] and [7].

The following result plays a key role in this paper.

Theorem 1. If $\{x(s, t),(s, t) \in Q\}$ is the standard Yeh-Wiener process, then the process $\{x(s, t)-[x](s, t),(s, t) \in Q\}$ and $X_{\tau}(x)=\left(x\left(s_{1}, t_{1}\right), \ldots, x\left(s_{m}, t_{n}\right)\right)$ are (stochastically) independent.

Proof. Using the expression (2.1), we may write for $(s, t)$ in $Q_{i j}$

$$
\begin{aligned}
x(s, t)-[x](s, t)= & x(s, t)-x\left(s_{i-1}, t_{j-1}\right) \\
& \left.-\left[\left(s-s_{i}\right)\left(t-t_{j}\right) / \Delta_{i} s \Delta_{j} t\right)\right] \Delta_{i j} x(s, t) \\
& -\left[\left(s-s_{i}\right) / \Delta_{i} s\right]\left(x\left(s_{i}, t_{j-1}\right)-x\left(s_{i-1}, t_{j-1}\right)\right) \\
& -\left[\left(t-t_{j}\right) / \Delta_{j} t\right]\left(x\left(s_{i-1}, t_{j}\right)-x\left(s_{i-1}, t_{j-1}\right)\right) .
\end{aligned}
$$

Thus, it suffices to show that $(2.3)$ is independent of $x\left(s_{p}, t_{q}\right)$ for $p=1, \ldots, m$ and $q=1, \ldots, n$. In view of equation (2.3) and repeated use of the formula

$$
E[x(s, t) x(u, v)]=\min \{s, u\} \min \{t, v\},
$$


a straghtforward computation yields

$$
E\left[x\left(s_{p}, t_{q}\right)\{x(s, t)-[x](s, t)\}\right]=0 .
$$

Because both $x\left(s_{p}, t_{q}\right)$ and $x(s, t)-[x](s, t)$ are Gaussian and uncorrelated, we may conclude that they are independent.

The following corollary is an immediate consequence of Theorem 1 .

Corollary 1.1. The two processes $\{x(s, t)-[x](s, t),(s, t) \in Q\}$ and $\{[x](s, t)$, $(s, t) \in Q\}$ are independent.

The following theorem plays an important role in the development of a simple formula for conditional Yeh-Wiener integrals.

Theorem 2. Let $F \in L_{1}\left(C(Q), m_{y}\right)$. Then for every $B \in \mathscr{B}^{m n}$,

$$
\int_{X_{\tau}^{-1}(B)} F(x) m_{y}(d x)=\int_{B} E[F(x-[x]+[\vec{\xi}])] P_{X_{\tau}}(d \vec{\xi}) .
$$

Proof. We first consider the case when $F$ is the indicator function of a YehWiener measurable set $M$, say $F(x)=I_{M}(x)$. Then

$$
\begin{aligned}
\int_{X_{\tau}^{-1}(B)} I_{M}(x) m_{y}(d x) & =m_{y}\left(M \cap X_{\tau}^{-1}(B)\right) \\
& =\int_{B} m_{y}\left(x \in M \mid X_{\tau}(x)=\vec{\xi}\right) P_{X_{\tau}}(d \vec{\xi}) \\
& =\int_{B} m_{y}\left(x-[x]+[\vec{\xi}] \in M \mid X_{\tau}(x)=\vec{\xi}\right) P_{X_{\tau}}(d \vec{\xi}) .
\end{aligned}
$$

But, as was shown in Theorem $1, x-[x]$ and $X_{\tau}(x)$ are independent, and thus so are $x-[x]+[\vec{\xi}]$ and $X_{\tau}(x)$. Therefore

$$
\begin{aligned}
\int_{X_{\tau}^{-1}(B)} I_{M}(x) m_{y}(d x) & =\int_{B} m_{y}(x-[x]+[\vec{\xi}] \in M) P_{X_{\tau}}(d \vec{\xi}) \\
& =\int_{B} E\left[I_{M}(x-[x]+[\vec{\xi}])\right] P_{X_{\tau}}(d \vec{\xi})
\end{aligned}
$$

Thus, (2.4) holds for the indicator function of any Yeh-Wiener measurable set. The general case follows by the usual arguments in integration theory.

From (1.2) and (2.4) we may conclude that for $F$ in $L_{1}\left(C(Q), m_{y}\right)$, $E\left(F(x) \mid X_{\tau}(x)=\vec{\xi}\right)$ and $E[F(x-[x]+[\vec{\xi}])]$ are equal for a.e. $\vec{\xi}$ in $\mathbf{R}^{m n}$. But while the former is Borel measurable by definition, the latter may only be Lebesgue measurable as the following example shows.

Example. Let $A$ be a Lebesgue measurable null set in $\mathbf{R}^{m n}$ that is not Borel measurable and let $M=\left\{x \in C(Q): X_{\tau}(x) \in A\right\}$. Then $m_{y}(M)=0$. Let $F(x)=I_{M}(x)$. Then $F(x)=I_{A}\left(X_{\tau}(x)\right)$ and

$$
\begin{aligned}
E[F(x-[x]+[\vec{\xi}])] & =E\left[I_{M}(x-[x]+[\vec{\xi}])\right] \\
& =E\left[I_{A}\left(X_{\tau}(x-[x]+[\vec{\xi}])\right)\right]=E\left[I_{A}(\vec{\xi})\right]=I_{A}(\vec{\xi})
\end{aligned}
$$


which is Lebesgue but not Borel measurable on $\mathbf{R}^{m n}$.

But if $f(\vec{\xi})$ is Lebesgue measurable on $\mathbf{R}^{m n}$, then there exists a Borel measurable function $\hat{f}(\vec{\xi})$, which is unique up to Borel null sets, such that $\hat{f}(\vec{\xi})=f(\vec{\xi})$ a.e. on $\mathbf{R}^{m n}$. Thus we may define:

Definition 1. If $F$ is Yeh-Wiener integrable on $C(Q)$, then by

$$
\widehat{E}[F(x-[x]+[\vec{\xi}])]
$$

we mean any Borel measurable function of $\vec{\xi}$ which is equal to

$$
E[F(x-[x]+[\vec{\xi}])
$$

for a.e. $\vec{\xi}$ in $\mathbf{R}^{m n}$.

Thus we have the following useful formula which is quite simple to apply in various applications.

Theorem 3. Let $F \in L_{1}\left(C(Q), m_{y}\right)$. Then

$$
E\left(F(x) \mid X_{\tau}(x)=\vec{\xi}\right)=\widehat{E}[F(x-[x]+[\vec{\xi}])] .
$$

In particular, if $F$ is Borel measurable, then

$$
E\left(F(x) \mid X_{\tau}(x)=\vec{\xi}\right)=E[F(x-[x]+[\vec{\xi}])] .
$$

The equalities in (2.5) and (2.6) mean that both sides are Borel measurable functions of $\vec{\xi}$ and they are equal except for Borel null sets.

\section{THREE EXAMPLES}

The following examples demonstrate that Theorem 3 is very useful and greatly simplies the calculations of conditional Yeh-Wiener integrals as compared to previous methods.

Example 1. For $x$ in $C(Q)$ let $F(x)=\int_{Q} x(s, t) d s d t=\int_{0}^{T} \int_{0}^{S} x(s, t) d s d t$. Then by (2.6) and (2.2)

$$
\begin{aligned}
& E\left(\int_{Q} x(s, t) d s d t \mid X_{\tau}(x)=\vec{\xi}\right) \\
& \quad=E\left[\int_{Q}(x(s, t)-[x](s, t)+[\vec{\xi}](s, t)) d s d t\right] \\
& \quad=\int_{Q} E[x(s, t)-[x](s, t)+[\vec{\xi}](s, t)] d s d t=\int_{Q}[\vec{\xi}](s, t) d s d t \\
& \quad=\sum_{i=1}^{m} \sum_{j=1}^{n} \frac{\Delta_{i} s \Delta_{j} t}{4}\left\{\xi_{i, j}+\xi_{i-1, j}+\xi_{i, j-1}+\xi_{i-1, j-1}\right\} .
\end{aligned}
$$

In particular, if $m=n=1$, then

$$
E\left(\int_{0}^{T} \int_{0}^{S} x(s, t) d s d t \mid X(S, T)=\xi\right)=\frac{1}{4} \xi S T .
$$

The conditional Yeh-Wiener integral in (3.2) was computed by Chung and Ahn [4] by a different method, and the computation was rather lengthy. 
Example 2. For $x \in C(Q)$ let $F(x)=\int_{Q} x^{2}(s, t) d s d t$. Then by (2.6)

$$
\begin{gathered}
E\left(\int_{Q} x^{2}(s, t) d s d t \mid X_{\tau}(x)=\vec{\xi}\right)=E\left[\int_{Q}(x(s, t)-[x](s, t)+[\vec{\xi}](s, t))^{2} d s d t\right] \\
=\int_{Q} E\left[(x(s, t)-[x](s, t))^{2}+([\vec{\xi}](s, t))^{2}\right. \\
\quad-2[\vec{\xi}](s, t)(x(s, t)-[x](s, t))] d s d t .
\end{gathered}
$$

As $x-[x]$ and $[x]$ are independent on $Q$ by Corollary 1.1,

$$
E[(x(s, t)-[x](s, t))[x](s, t)]=0,
$$

and hence by the use of (2.1) with the formula

$$
\begin{gathered}
E[x(s, t) x(u, v)]=\min \{s, u\} \min \{t, v\}, \\
\int_{Q} E[(x(s, t)-[x](x, t))]^{2} d s d t=\int_{Q} E[x(s, t)(x(s, t)-[x](s, t))] d s d t \\
=\int_{Q} s t d s d t-\sum_{i=1}^{m} \sum_{j=1}^{n} \int_{Q_{i j}}\left\{\begin{array}{c}
s_{i-1} t_{j-1}+\frac{\left(s-s_{i-1}\right)^{2}\left(t-t_{j-1}\right)^{2}}{\Delta_{i} s \Delta_{j} t} \\
\left.+\frac{t_{j-1}\left(s-s_{i-1}\right)^{2}}{\Delta_{i} s}+\frac{s_{i-1}\left(t-t_{j-1}\right)^{2}}{\Delta_{j} t}\right\} d s d t \\
=\frac{S^{2} T^{2}}{4}-\sum_{i=1}^{m} \sum_{j=1}^{n}\left\{s_{i-1} t_{j-1} \Delta_{i} s \Delta_{j} t+\frac{1}{9}\left(\Delta_{i} s\right)^{2}\left(\Delta_{j} t\right)^{2}\right. \\
\left.+\frac{1}{3} t_{j-1}\left(\Delta_{i} s\right)^{2} \Delta_{j} t+\frac{1}{3} s_{i-1} \Delta_{i} s\left(\Delta_{j} t\right)^{2}\right\} \\
=\frac{S^{2} T^{2}}{4}-\frac{1}{9}\left\{\sum_{i=1}^{m} \Delta_{i} s\left(s_{i}+2 s_{i-1}\right)\right\}\left\{\sum_{j=1}^{n} \Delta_{j} t\left(t_{j}+2 t_{j-1}\right)\right\} .
\end{array}\right.
\end{gathered}
$$

Thus,

$$
\begin{aligned}
& \left.E\left(\int_{Q} x^{2}(s, t) d s d t \mid X_{\tau}(x)=\vec{\xi}\right)\right) \\
= & \frac{s^{2} T^{2}}{4}+\int_{Q}([\vec{\xi}](s, t))^{2} d s d t \\
& -\frac{1}{9}\left\{\sum_{i=1}^{m} \Delta_{i} s\left(s_{i}+2 s_{i-1}\right)\right\}\left\{\sum_{j=1}^{n} \Delta_{j} t\left(t_{j}+2 t_{j-1}\right)\right\} .
\end{aligned}
$$

In particular, if $m=n=1$, then

$$
\begin{aligned}
& \left.E\left(\int_{0}^{T} \int_{0}^{S} x^{2}(s, t) d s d t \mid x(S, T)=\xi\right)\right) \\
& \quad=\frac{S^{2} T^{2}}{4}+\int_{Q}\left(\frac{s t \xi}{S T}\right)^{2} d s d t-\frac{1}{9} S^{2} T^{2}=\frac{5}{36}(S T)^{2}+\frac{1}{9} S T \xi^{2}
\end{aligned}
$$

which agrees with Chung and Ahn's computation in [4]. 
Example 3. For $x \in C(Q)$ let $F(x)=\exp \left\{\int_{Q} x(s, t) d s d t\right\}$. Then using (2.6) we see that

$$
\begin{aligned}
E(\exp & \left.\left\{\int_{Q} x(s, t) d s d t\right\} \mid X_{\tau}(x)=\vec{\xi}\right) \\
= & E\left[\exp \left\{\int_{Q}(x(s, t)-[x](s, t)+[\vec{\xi}](s, t)) d s d t\right\}\right] \\
= & \exp \left\{\int_{Q}[\vec{\xi}](s, t) d s d t\right\} E\left[\exp \left\{\int_{Q}(x(s, t)-[x](s, t) d s d t\}\right] .\right.
\end{aligned}
$$

In particular, for each fixed $u \in C(Q)$,

$$
\begin{aligned}
& E\left(\exp \left\{\int_{Q} x(s, t) d s d t\right\} \mid X_{\tau}(x)=X_{\tau}(u)\right) \\
& \quad=\exp \left\{\int_{Q}[u](s, t) d s d t\right\} E\left[\exp \left\{\int_{Q}(x(s, t)-[x](s, t)) d s d t\right\}\right] .
\end{aligned}
$$

Thus

$$
\lim _{\|\tau\| \rightarrow 0} E\left(\exp \left\{\int_{Q} x(s, t) d s d t\right\} \mid X_{\tau}(x)=X_{\tau}(u)\right)=\exp \left\{\int_{Q} u(s, t) d s d t\right\}
$$

as expected.

\section{Conditional EXPECTATION OF FUNCTIONS INVOLVING STOCHASTIC INTEGRALS.}

In the next section we will be translating $x(s, t)$ by the function $x_{0}(s, t)=$ $\int_{0}^{t} \int_{0}^{s} h(u, v) d u d v$ for fixed $h \in L_{2}(Q)$. It turns out that using $\tilde{h}$, the sectional average of $h$, simplies both the statement and the proof of the conditional translation theorem.

Definition 2. Let $\tau=\left\{\left(s_{i}, t_{j}\right), i=1, \ldots, m\right.$ and $\left.j=1, \ldots, n\right\}$ be a partition of $Q$. Then for each function $h \in L_{2}(Q)$, we define the sectional average of $h$ by

$$
\tilde{h}(s, t)=\frac{1}{\Delta_{i} s \Delta_{j} t} \int_{Q_{i j}} h(u, v) d u d v
$$

on each $Q_{i j}=\left(s_{i-1}, s_{i}\right] \times\left(t_{j-1}, t_{j}\right]$ and $\tilde{h}(s, t)=0$ if $s t=0$.

The following theorem gives an interesting relationship involving $\tilde{h}$ and $[x]$ that is very useful in computing conditional expectations.

Theorem 4. Let $h \in L_{2}(Q)$. Then

$$
\begin{aligned}
\int_{Q} h(s, t) \tilde{h}(s, t) d s d t & =\int_{Q} \tilde{h}^{2}(s, t) d s d t \\
\|h-\tilde{h}\|_{2}^{2} & =\|h\|_{2}^{2}-\|\tilde{h}\|_{2}^{2} \geq 0, \text { and } \\
\int_{Q} h d[x] & =\int_{Q} \tilde{h} d x=\int_{Q} \tilde{h} d[x] \text { for each } x \in C(Q) .
\end{aligned}
$$


Proof. (4.1) follows easily from the definition of $\tilde{h}$ while (4.2) follows from (4.1). We note that each of the integrals in (4.3) exists since $\tilde{h}$ is of bounded variation on $Q$ and $[x]$ is absolutely continuous on $Q$ with $\partial^{2}[x](s, t) / \partial s \partial t$ in $L_{2}(Q)$. Next, using (2.1) we see that for each $Q_{i j}$,

$$
\int_{Q_{i j}} h d[x]=\frac{\Delta_{i j} x(s, t)}{\Delta_{i} s \Delta_{j} t} \int_{Q_{i j}} h(s, t) d s d t=\Delta_{i j} x(s, t) \tilde{h}\left(s_{i}, t_{j}\right)
$$

from which (4.3) follows immediately.

Theorem 5. Let $h_{1}, \ldots, h_{N} \in L_{2}(Q)$ and let $g_{j}=h_{j}-\tilde{h}_{j}$ for $j=1, \ldots, N$. If $F\left(\int_{Q} h_{1} d x, \ldots, \int_{Q} h_{N} d x\right)$ is Yeh-Wiener integrable, then

$$
\begin{aligned}
E\left(F\left(\int_{Q} g_{1} d x, \ldots, \int_{Q} g_{N} d x\right)\right. & \left.\mid X_{\tau}(x)=\vec{\xi}\right) \\
= & E\left[F\left(\int_{Q} g_{1} d x, \ldots, \int_{Q} g_{N} d x\right)\right] .
\end{aligned}
$$

Furthermore, if $\left\{\alpha_{1}, \ldots, \alpha_{k}\right\}$ is a set of orthogonal functions on $Q$ with $\operatorname{span}\left\{\alpha_{1}, \ldots, \alpha_{k}\right\}=\operatorname{span}\left\{g_{1}, \ldots, g_{N}\right\}$, then there exists a function $G$ such that

$$
F\left(\int_{Q} g_{1} d x, \ldots, \int_{Q} g_{N} d x\right)=G\left(\int_{Q} \alpha_{1} d x, \ldots, \int_{Q} \alpha_{k} d x\right)
$$

and so

$$
\begin{aligned}
E & \left(F\left(\int_{Q} g_{1} d x, \ldots, \int_{Q} g_{N} d x\right) \mid X_{\tau}(x)=\vec{\xi}\right) \\
& =\prod_{j=1}^{k}\left(2 \pi\left\|\alpha_{j}\right\|^{2}\right)^{-1 / 2} \int_{\mathbf{R}^{k}} G\left(v_{1}, \ldots, v_{k}\right) \exp \left\{-\frac{1}{2} \sum_{j=1}^{k} \frac{v_{j}^{2}}{\left\|\alpha_{j}\right\|^{2}}\right\} d v_{1} \ldots d v_{k} .
\end{aligned}
$$

Proof. By Theorem 3,

$$
\begin{aligned}
E( & \left.F\left(\int_{Q} g_{1} d x, \ldots, \int_{Q} g_{N} d x\right) \mid X_{\tau}(x)=\vec{\xi}\right) \\
& \left.=\widehat{E}\left[F\left(\int_{Q} g_{1} d(x-[x]+[\vec{\xi}])\right), \ldots, \int_{Q} g_{N} d(x-[x]+[\vec{\xi}])\right)\right] .
\end{aligned}
$$

But, by use of (4.3),

$$
\begin{aligned}
\int_{Q} g_{j} d(x-[x]+[\vec{\xi}]) & =\int_{Q} g_{j} d x-\int_{Q} g_{j} d[x]+\int_{Q} g_{j} d[\vec{\xi}] \\
& =\int_{Q} g_{j} d x-\int_{Q} \tilde{g}_{j} d x+\int_{Q} \tilde{g}_{j} d[\vec{\xi}]
\end{aligned}
$$

However $g_{j}=h_{j}-\tilde{h}_{j}$ and so $\tilde{g}_{j}=\tilde{h}_{j}-\tilde{h}_{j}=0$. Thus

$$
\int_{Q} g_{j} d(x-[x]+[\vec{\xi}])=\int_{Q} g_{j} d x
$$


from which (4.4) readily follows. Equations (4.5) and (4.6) are obvious.

Corollary 5.1. Let $h_{1}, \ldots, h_{N} \in L_{2}(Q)$ satisfy the condition $\int_{Q} h_{j}(s, t) d s d t=0$ for $j=1, \ldots, N$. Then

$$
\begin{gathered}
E\left(F\left(\int_{Q} h_{1} d x, \ldots, \int_{Q} h_{N} d x\right) \mid x(S, T)=\xi\right) \\
=E\left[F\left(\int_{Q} h_{1} d x, \ldots, \int_{Q} h_{N} d x\right)\right]
\end{gathered}
$$

which can be evaluated using (4.6).

Proof. Since $\tau$ consists of the single point $(S, T)$, it follows that $\tilde{h}_{j}(s, t)=$ $\frac{1}{S T} \int_{Q} h_{j}(u, v) d u d v=0$ for $j=1, \ldots, N$. Hence $g_{j}=h_{j}$ for all $j$, and so the result follows from Theorem 5 .

Theorem 6. Let $h \in L_{2}(Q)$ and assume that $F\left(\int_{Q} h d x\right)$ is Yeh-Wiener integrable. Then

$$
\begin{aligned}
& E\left(F\left(\int_{Q} h d x\right) \mid X_{\tau}(x)=\vec{\xi}\right) \\
& \quad=\frac{1}{\sqrt{2 \pi}\|h-\tilde{h}\|} \int_{-\infty}^{\infty} F(v) \exp \left\{\frac{-\left(v-\int_{Q} h d[\vec{\xi}]\right)^{2}}{2\|h-\tilde{h}\|^{2}}\right\} d v .
\end{aligned}
$$

Proof. By Theorem 3 and (4.3) we have

$$
\begin{aligned}
E( & \left.F\left(\int_{Q} h d x\right) \mid X_{\tau}(x)=\vec{\xi}\right)=\widehat{E}\left[F\left(\int_{Q} h d(x-[x]+[\vec{\xi}])\right)\right] \\
= & \widehat{E}\left[F\left(\int_{Q}(h-\tilde{h}) d x+\int_{Q} h d[\vec{\xi}]\right)\right] \\
= & (2 \pi)^{-1 / 2} \int_{-\infty}^{\infty} F\left(\|h-\tilde{h}\| u+\int_{Q} h d[\vec{\xi}]\right) \exp \left\{-\frac{u^{2}}{2}\right\} d u .
\end{aligned}
$$

\section{TRANSLATION OF CONDITIONAL YeH-WiENER INTEGRALS}

The Cameron-Martin translation theorem in Yeh-Wiener space $C(Q)$ (see [11]) states that if $x_{0}(s, t)=\int_{0}^{t} \int_{0}^{s} h(u, v) d u d v$ for some $h \in L_{2}(Q)$, and if $T_{1}$ is the translation of $C(Q)$ into itself given by $z=T_{1}(x)=x+x_{0}$, then for any Yeh-Wiener integrable functional $F$ on $C(Q)$ and any Yeh-Wiener measurable set $\Gamma$

$$
\int_{\Gamma} F(z) m_{y}(d z)=\int_{T_{1}^{-1}(\Gamma)} F\left(x+x_{0}\right) J\left(x_{0}, x\right) m_{y}(d x),
$$

where

$$
J\left(x_{0}, x\right)=\exp \left\{-\frac{1}{2} \int_{Q} h^{2}(s, t) d s d t\right\} \exp \left\{-\int_{Q} h(s, t) d x(s, t)\right\}
$$


and $\int_{Q} h(s, t) d x(s, t)$ is a stochastic integral with mean zero and variance $\int_{Q} h^{2}(s, t) d s d t$. In particular, if $\Gamma=C(Q)$, then (5.1) becomes

$$
E[F(z)]=E\left[F\left(x+x_{0}\right) J\left(x_{0}, x\right)\right] .
$$

The following is the vector-valued conditional version of (5.3).

Theorem 7. Let $x_{0}(s, t)=\int_{0}^{t} \int_{0}^{s} h(u, v) d u d v$ on $Q$ for some $h \in L_{2}(Q)$. Let $F \in L_{1}\left(C(Q), m_{y}\right)$. Then for each partition $\tau=\left\{\left(s_{i}, t_{j}\right), i=1, \ldots, m, j=\right.$ $1, \ldots, n\}$ of $Q$,

$$
\begin{aligned}
& E\left(F(z) \mid X_{\tau}(z)=\vec{\xi}\right) \\
&= E\left(F\left(x+x_{0}\right) J\left(x_{0}, x\right) \mid X_{\tau}\left(x+x_{0}\right)=\vec{\xi}\right) \\
& \cdot \exp \left\{-\frac{1}{2} \int_{Q} \tilde{h}^{2}(s, t) d s d t+\int_{Q} h(s, t) d[\vec{\xi}](s, t)\right\} .
\end{aligned}
$$

Proof. By Theorem 3, we have

$$
E\left(F(z) \mid X_{\tau}(z)=\vec{\xi}\right)=\widehat{E}[F(z-[z]+[\vec{\xi}])] .
$$

Noting that $\left[x+x_{0}\right](s, t)=[x](s, t)+\left[x_{0}\right](s, t)$, and using (5.3) we see that

$$
E[F(z-[z]+[\vec{\xi}])]=E\left[F\left(x+x_{0}-[x]-\left[x_{0}\right]+[\vec{\xi}]\right) J\left(x_{0}, x\right)\right] .
$$

Next we rewrite $J\left(x_{0}, x\right)$ in the form

$$
\begin{aligned}
J\left(x_{0}, x\right)= & \exp \left\{-\frac{1}{2} \int_{Q} h^{2}(s, t) d s d t\right\} \exp \left\{-\int_{Q} h d\left(x-[x]+[\vec{\xi}]-\left[x_{0}\right]\right)\right\} \\
& \exp \left\{-\int_{Q} h d[x]\right\} \exp \left\{\int_{Q} h d\left([\vec{\xi}]-\left[x_{0}\right]\right)\right\} .
\end{aligned}
$$

Since $x-[x]$ and $[x]$ are two independent processes on $Q$ by Corollary 1.1, it follows from (5.5) and (5.6) that

$$
\begin{aligned}
& E[F(z-[z]+[\vec{\xi}])] \\
= & E\left[F\left(x+x_{0}-[x]-\left[x_{0}\right]+[\vec{\xi}]\right) \exp \left\{-\int_{Q} h d\left(x-[x]+[\vec{\xi}]-\left[x_{0}\right]\right)\right\}\right] \\
\cdot & E\left[\exp \left\{-\int_{Q} h d[x]\right\}\right] \exp \left\{-\frac{1}{2} \int_{Q} h^{2}(s, t) d s d t+\int_{Q} h d\left([\vec{\xi}]-\left[x_{0}\right]\right)\right\} .
\end{aligned}
$$

Since $\int_{Q} \tilde{h} d x$ is a Gaussian random variable with mean zero and variance $\|\tilde{h}\|_{2}^{2}$ it follows using (4.3) that

$$
E\left[\exp \left\{-\int_{Q} h d[x]\right\}\right]=E\left[\exp \left\{-\int_{Q} \tilde{h} d x\right\}\right]=\exp \left\{\frac{1}{2} \int_{Q} \tilde{h}^{2}(s, t) d s d t\right\} .
$$


Next using Theorem 4 we have

$$
\begin{aligned}
\int_{Q} h d\left[x_{0}\right] & =\int_{Q} \tilde{h} d x_{0}=\int_{Q} \tilde{h}(s, t) \frac{\partial^{2} x_{0}(s, t)}{\partial s \partial t} d s d t \\
& =\int_{Q} \tilde{h}(s, t) h(s, t) d s d t=\int_{Q} \tilde{h}^{2}(s, t) d s d t .
\end{aligned}
$$

Upon applying (2.5), (5.8), and (5.9) to (5.7), we obtain

$$
\begin{aligned}
\widehat{E}[F(z-[z]+[\vec{\xi}])] & \\
= & E\left(F\left(x+x_{0}\right) J\left(x_{0}, x\right) \mid X_{\tau}\left(x+x_{0}\right)=\vec{\xi}\right) \\
& \cdot \exp \left\{\frac{1}{2} \int_{Q} \tilde{h}^{2}(s, t) d s d t\right\} \exp \left\{\int_{Q} h d[\vec{\xi}]-\int_{Q} \tilde{h}^{2}(s, t) d s d t\right\} .
\end{aligned}
$$

This together with (5.4) gives the desired result.

Note that if the partition $\tau$ consists of the single point $(S, T)$, then Theorem 7 reduces to the following corollary:

Corollary 7.1. Let $F, h$ and $x_{0}$ be as in Theorem 7. Then

$$
\begin{aligned}
E(F(z) \mid z(S, T)=\xi) & =E\left(F\left(x+x_{0}\right) J\left(x_{0}, x\right) \mid x(S, T)=\xi-x_{0}(S, T)\right) \\
& \exp \left\{-\frac{x_{0}^{2}(S, T)}{2 S T}+\frac{\xi x_{0}(S, T)}{S, T}\right\} .
\end{aligned}
$$

This corollary was the main result in Chang and Ahn [2]; however their method was very different than ours.

By choosing $F(x) \equiv 1$ in Theorem 7 we obtain the following corollary:

Corollary 7.2. Let $h$ and $x_{0}$ be as in Theorem 7. Then

$$
\begin{aligned}
& E\left(\exp \left\{\int_{Q} h(s, t) d x(s, t)\right\} \mid X_{\tau}(x)=\tilde{\eta}\right) \\
& =\exp \left\{\frac{1}{2} \int_{Q}\left(h^{2}(s, t)-\tilde{h}^{2}(s, t)\right) d s d t+\int_{Q} h d[\tilde{\eta}]\right\} .
\end{aligned}
$$

Proof. By setting $F \equiv 1$ and $\xi_{i, j}=\eta_{i, j}+x_{0}\left(s_{i}, t_{j}\right) \forall\left(s_{i}, t_{j}\right) \in \tau$ in Theorem 7, we obtain

$$
1=E\left(J\left(x_{0}, x\right) \mid X_{\tau}(x)=\ddot{\eta}\right) \exp \left\{-\frac{1}{2} \int_{Q} \tilde{h}^{2}(s, t) d s d t+\int_{Q} h d[\tilde{\eta}]+\int_{Q} h d\left[x_{0}\right]\right\} .
$$

Using (5.9) in (5.10) and using (5.2), we get that

$$
\begin{aligned}
E\left(\exp \left\{-\int_{Q} h(s, t) d x(s, t)\right\} \mid X_{\tau}(x)=\vec{\eta}\right) \\
=\exp \left\{\frac{1}{2} \int_{Q}\left(h^{2}(s, t)-\tilde{h}^{2}(s, t)\right) d s d t-\int_{Q} h d[\tilde{\eta}]\right\} .
\end{aligned}
$$

Thus, the result readily follows by replacing $h(s, t)$ by $-h(s, t)$.

Remark. Corollary 7.2 is also a special case of Theorem 6. 


\section{REFERENCES}

1. L. Breiman, Probability, Addision Wesley, Reading, Mass., 1968.

2. K. S. Chang and J. M. Ahn, Translation theorem for conditional Yeh-Wiener integrals, J. Korean Math. Soc., 20 (1983), 109-118.

3. K. S. Chang, J. M. Ahn and J. S. Chang, An evaluation of the conditional Yeh-Wiener integral, Pacific J. Math., 124 (1986), 107-117.

4. D. M. Chung and J. M. Ahn, Conditional Yeh-Wiener integrals, J. Korean Math. Soc., 20 (1983), 209-221.

5. J. L. Doob, Stochastic processes, Wiley, New York, 1965.

6. G. W. Johnson and D. L. Skoug, A stochastic integration formula for two-parameter Wiener $\times$ two-parameter Wiener space, Siam J. Math. Anal., 18 (1987), 919-932.

7. C. Park, On Fredholm transformations in Yeh-Wiener space, Pacific J. Math., 40 (1972), 173195.

8. C. Park and D. L. Skoug, A simple formula for conditional Wiener integrals with applications, Pacific J. Math., 134 (1988).

9. H. G. Tucker, A graduate course in probability, Academic Press, New York, 1967.

10. J. Yeh, Wiener measure in a space of functions of two variables, Trans. Amer. Math. Soc., 95 (1960), 433-450.

11. _ Cameron-Martin translation theorem in the Wiener space of functions of two variables, Trans. Amer. Math. Soc., 107 (1963), 409-420.

12. _ Stochastic processes and the Wiener integral, Marcel Dekker, New York, 1973.

13. _ Inversion of conditional Wiener integrals, Pacific J. Math., 59 (1975), 623-638.

14. _ Transformation of conditional Wiener integrals under translation and the CameronMartin translation theorem, Tohoku Math. J., 30 (1978), 505-515.

Department of Mathematics and Statistics, Miami University, Oxford, Ohio 45056

Department of Mathematics and Statistics, University of Nebraska, Lincoln, NeBRASKA 68588 